\title{
Erratum
}

\section{Synthetic Studies on the MARDi Cascade: Stereoselective Preparation of Sulfonyl-Substituted Seven-Membered Rings}

Yoann Coquerel,* David Bensa, Vincent Moret, Jean Rodriguez* Synlett 2006, 2751.

The structure of cycloheptanol $\mathbf{8}$ in Table 1 was incorrectly represented. The correct structure is:

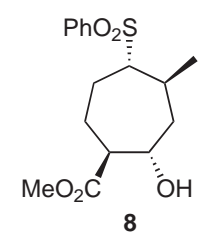

